Elektrokonvulsionstherapie unter Zwang? Kontra

Die Elektrokrampftherapie (EKT) ist weltweit ein anerkanntes Therapieverfahren, welches in den westlichen Industrieländern im Wesentlichen bei depressiven Patienten eingesetzt wird. Insgesamt ist die Domäne der EKT die Behandlung von therapieresistenten Depressionen [1]. Die EKT ist dabei das wirkstärkste antidepressive Behandlungsverfahren. Die EKT hat in den letzten 10-20 Jahren wieder eine zunehmende Bedeutung im klinischen Alltag gefunden und auch in Deutschland hat die Anzahl der mit EKT behandelten Patienten zugenommen. Die Anzahl der in Deutschland mit EKT behandelten Patienten wird aktuell auf etwa 5000 Patienten pro Jahr geschätzt. Die Elektrokrampftherapie hat nach ihrer Einführung im Jahre 1938 (erste Behandlung in der römischen Universitätsklinik) Phasen von hoher Akzeptanz und auch von deutlicher Ablehnung erlebt. Insbesondere nach dem Zweiten Weltkrieg vor Einführung der Antidepressiva - war EKT lange Zeit der Goldstandard in der Behandlung der Depression. In den 1970er-Jahren gab es zum Teil sehr scharfe und polemische Debatten um EKT. Die damalige Kritik an der Durchführung der EKT war nicht vollständig unberechtigt; so gab es Defizite in der Indikationsstellung und bei der sachgerechten Durchführung der Elektrokrampftherapie. In Deutschland galt es bei einem nicht unbeträchtlichen Teil von psychiatrischen Kliniken geradezu als fortschrittlich, auf EKT zu verzichten, die in Anteilen - wenn auch fälschlich als Ausdruck einer „repressiven Psychiatrie“ angesehen wurde. In dieser Zeit entstand z. B. auch in den USA der Film „Einer flog über das Kuckucksnest“ mit Jack Nicolson. Letztendlich hat diese Debatte dazu geführt, dass die APA unter Federführung von Max Fink [2] (New York) Guidelines zur Durchführung der EKT formulierte. Die Zeiten von geradezu polemischen Debatten um die EKT sind in meiner Wahrnehmung vergangen; dies ändert aber nichts daran, dass es gleichwohl anhaltend Vorbehalte gegenüber der EKT gibt. Diese Vorbehalte speisen sich aus mehreren Quellen.

Hierzu gehören zum einen die historischen Wurzeln der EKT; die EKT wurde bis in die 1950er-Jahre häufig ohne Narkose durchgeführt und deshalb war die EKT deutlich nebenwirkungsträchtiger. Es wurden mit EKT Patienten behandelt, die heutzutage in keinem Fall mit einer EKT behandelt würden, z. B. Patienten mit chronischen schizophrenen Psychosen [3]. Auch Fragen der Zustimmung („informed consent“) spielten in der ferneren Vergangenheit bei der Anwendung der EKT keine Rolle; das hat sich allerdings nun schon seit geraumer Zeit gemäß den üblichen rechtlichen Standards verändert.

- Im Regelfall kommen Patienten zu uns, damit sie EKT bekommen und sie geben nach entsprechender Aufklärung dafür ihre informierte Zustimmung. Diese Patienten müssen nicht gegen ihren Willen mit EKT behandelt werden, sondern sie haben sich ausdrücklich für EKT als Option entschieden. Wir müssen meiner Einschätzung nach alles dafür tun, die bereits deutlich verbesserte Akzeptanz der Elektrokrampftherapie weiter zu steigern. Die Evolution der EKT der letzten Jahre ist noch nicht an ihrem Endpunkt angekommen. Dies gilt für das eigentliche Behandlungsprozedere, die Technik und auch die Narkoseverfahren. Zusätzlich sollten wir uns als Behandler darum bemühen, es unseren $\mathrm{Pa}$ tienten so leicht wie möglich zu machen, die Zustimmung zur EKT zu geben. Auch die Anwesenheit von Angehörigen bei der EKT-Behandlung (!) kann die Akzeptanz verbessern [4]. Seit wenigen Jahren ist die Teilnahme von Angehörigen bei der EKT unter gewissen Umständen bei uns möglich.
Aus meiner Perspektive müssen gerade wir Psychiater akzeptieren, dass Behandlungen gegen den ausdrücklichen Wunsch eines Patienten im Regelfall nicht durchgeführt werden können. Die Psychiatrie steht immer wieder im Verdacht, dass Behandlungen (welcher Art auch immer) erzwungen würden. Dies schadet unserer Disziplin, aber letztendlich auch unseren Patienten. Auch wir Psychiater müssen damit leben, dass Patienten sinnvolle Behandlungen - wie z. B. die EKT - ablehnen.

- Die EKT ist zwar eine sehr verträgliche, erfolgreiche und im Regelfall auch sichere Behandlungsform; die Häufigkeit von schwerwiegenden Nebenwirkungen beträgt 1:500001:75000 Behandlungen. Durch technische Modifikationen (insbes. Kurzpulstechnik, Ultrakurzpulstechnik) ist die Häufigkeit von (vorübergehenden) kognitiven Nebenwirkungen zwar reduziert, aber solche Nebenwirkungen können gleichwohl vorübergehend auftreten. Diese - wenn auch vorübergehenden - Nebenwirkungen können bei Patienten (und Angehörigen) erhebliche Ängste auslösen und Vorbehalte in der Umgebung gegenüber der EKT auslösen.

- Die Vorstellung, dass ein Patient, der ausdrücklich EKT ablehnt, womöglich fixiert und schreiend über den Flur zum EKT-Behandlungsraum gebracht wird, ist für mich höchst aversiv. Eine solche Situation möchte ich nicht erleben.

- Unter bestimmten Umständen kann auch die Frage auftauchen, inwieweit ein Patient noch zustimmungsfähig ist. Wenn ein nicht mehr zustimmungsfähiger Patient (z. B. mit wahnhafter Depression) mit entsprechender Genehmigung durch den gesetzlichen Betreuer eine EKT-Behandlung bekommen soll und der Patient dies toleriert, ist dies für mich akzeptabel. 
Sofern eine Zustimmung zur Behandlung durch den gesetzlichen Betreuer vorliegt und der Patient aber die Behandlung ausdrücklich ablehnt, würde ich im Regelfall die Durchführung der EKT ablehnen. Zu Recht erwartet die Gesellschaft von uns Psychiatern, dass wir mit unseren Patienten (gegebenenfalls unter Einschaltung von Bezugspersonen) vorgesehene Behandlungsmaßnahmen thematisieren und uns äußerste Mühe geben, eine Zustimmung oder zumindest Akzeptanz der vorgesehenen Behandlungsmaßnahmen zu erreichen.

- Die EKT ist zwar in vielen Situationen eine höchst sinnvolle Behandlung, aber zumeist auch nicht alternativlos. Bei den sehr wenigen akut lebensbedrohlichen Situationen mit einer Notfallindikation zur EKT (z. B. maligne Katatonie [5]) ist der Patient im Regelfall nicht zustimmungsfähig (Genehmigung durch den rechtlichen Betreuer) und kann auch keine klaren Willensäußerungen abgeben, sodass es sich zwar um eine Behandlung ohne, aber nicht gegen den Willen handelt.
- Wir sollten auf Zwangsbehandlung mit EKT gegen den ausdrücklich geäußerten Willen des Patienten verzichten, auch wenn man von Einwilligungsunfähigkeit im juristischen Sinne ausgehen kann. Eine solche Grundeinstellung steigert letztendlich die Akzeptanz der EKT.

\section{Über die Autoren}

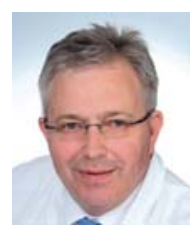

Here W. Folkerts

\section{Korrespondenzadresse}

Prof. Dr. med. Here W. Folkerts Chefarzt der Klinik für Psychiatrie, Psychotherapie und Psychosomatik Wilhelmshaven, Akademisches Lehrkrankenhaus der Universität Göttingen Friedrich-Paffrath-Straße 100 26389 Wilhelmshaven

E-Mail: here.folkerts@klinikum-whv.de

\section{Literatur}

[1] Folkerts HW. Elektrokrampftherapie. Indikation, Durchführung und Behandlungsergebnisse. Nervenarzt 2011; 82: 93-102

[2] Fink M. Convulsive Therapy: Theory and Practice. New York: Raven Press; 1979

[3] Fink M. Electroshock - Restoring the Mind. New York, Oxford: Oxford University Press; 1999

[4] Coffey M], Coffey CE. Patient-Centered Electroconvulsive Therapy Care: A Call to Action. J ECT 2016; 32: 78 - 79

[5] Shenai N, White CD, Azzam PN et al. Practical and Legal Challenges to Electroconvulsive Therapy in Malignant Catatonia. Harv Rev Psychiatry 2016; 24: 238 - 241

\section{Bibliografie}

DOI https://doi.org/10.1055/s-0043116926

Psychiat Prax 2017; 44: 314-315

(c) Georg Thieme Verlag KG

Stuttgart · New York

ISSN 0303-4259 